\title{
Screening of Cold Tolerant Rice Genotypes for Seedling Traits under Low Temperature Regimes
}

\author{
N. Srikanth Rahul ${ }^{1}$, D. Bhadru ${ }^{2}$, M. Sreedhar ${ }^{3}$ and S. Vanisri ${ }^{4 *}$ \\ ${ }^{1}$ Deptartment of Genetics and Plant Breeding, College of Agriculture, Professor Jayashankar \\ Telangana State Agricultural University, Hyderabad-500 030, Telangana, India \\ ${ }^{2}$ Seed Research and Technology Center, Rajendranagar, Hyderabad, Telangana, India \\ ${ }^{3}$ Quality Control Lab, Prof. Jayashankar Telangana State Agricultural University, Rajendranagar, \\ Hyderabad-500 030, Telangana, India \\ ${ }^{4}$ Institute of Biotechnology, Prof. Jayashankar Telangana State Agricultural University, \\ Rajendranagar, Hyderabad-500 030, Telangana, India \\ *Corresponding author
}

\section{A B S T R A C T}

Thirty seven rice genotypes with reported cold tolerance at various phonological stages were used to evaluate for germination and seedling traits in laboratory conditions under three temperature regimes viz., normal $\left(28^{\circ} \mathrm{C}\right), 8^{\circ} \mathrm{C}$ and $13^{\circ} \mathrm{C}$ respectively for

\section{Keywords}

Rice, Seedling cold tolerance, Rabi.

Article Info

Accepted:

28 November 2017

Available Online:

10 December 2017 identification of genotypes possessing seedling stage cold tolerance. The per se performance in cold environment was found to be related to parameters like germination percent, germination Index, root, shoot and seedling length and seedling vigour. In this context, it is very crucial to have a better understanding of the mechanism of cold tolerance at seedling stage, which is a potential production constraint in rabi. The response of genotypes differed with different low temperatures and was significant indicating the presence of considerable variability for cold tolerance at germination and seedling stage. Certain genotypes performed very well under $13^{\circ} \mathrm{C}$ and $8^{\circ} \mathrm{C}$ of temperatures for various seedling traits. However, few genotypes exhibited superior performance under $13^{\circ} \mathrm{C}$ but not performed well under $8^{\circ} \mathrm{C}$ of temperatures vice versa. On an overall basis HPR 1068, Himalaya-2216 and Vivek Dhan -82 showed lesser percentage of reduction for seedling vigour index at both temperatures and these genotypes can be used as cold tolerant donors in future breeding programs for development of seedling cold tolerant rice varieties.

\section{Introduction}

Climate change is likely to have a significant impact on agriculture, especially through the increased likelihood of extreme temperatures. Low temperature damage has long been a problem and has caused serious yield losses in more than 20 countries. The frequency of yield loss due to low temperature damage, vary from country to country and also region to region within the states. Rice is very sensitive to prolonged exposure to lower temperatures. Cold tolerance at seedling stage is a primary requirement of rice cultivars as seedlings are raised during the cold months of November and December even up to January (Tiwari et al., 2009). Low temperature during seedling stage causes poor germination, 
stunted seedling growth and seedling mortality which directly affect the yield (Pathak et al., 2003). The stunted seedling growth also affects manual uprooting and transplanting and further field establishment. The rice species (Oryza sativa L.) has wide adaptability to cold and cold-tolerant ecotypes are available for breeding. Japonica genotypes show higher cold tolerance at the germination stage than indica genotypes, although variability for this trait within both subspecies has been reported (Cruz and Milach, 2013). Evaluation of rice genotypes for represent traits under more than one temperature is necessary to distinguish cold tolerance. The availability of low temperature tolerant source is interesting for breeding cold tolerance, since it allows them to be used as progenitors in crosses as well as to transfer of desirable characters to make them adaptable to the target environment.

Keeping in view the above facts, an experiment was conducted in laboratory conditions simulating low field temperatures to evaluate rice genotypes against low temperature stress at germination stage for identification of the superior genotypes with good germination and seedling vigour traits. Identified genotypes can be utilized in future breeding programs for development of cold tolerant rice varieties at seedling stage.

\section{Materials and Methods}

Thirty seven rice genotypes collected from the VPKS, Almora, RRS, Palampur and SKAUT, Kashmir along with important cold tolerant varieties of ANGRAU (presently PJTSAU), Hyderabad are the basic material for the study. The experiment was carried out at Quality control Lab, PJTSAU, Rajendranagar, Hyderabad under the controlled conditions. All the genotypes were allowed to germinate at $28^{\circ} \mathrm{C}$ for seven days (control), $13^{\circ} \mathrm{C}$ and $8^{\circ} \mathrm{C}$ for 28 days (cold) as described by Cruz and Milach (2004). Twenty seeds of each genotype were placed in petri dishes containing 2 layers of germination paper, soaked with distilled water. Before placing the seeds for germination all the seeds were washed with aqueous ethanol $(70 \%$ $\mathrm{V} / \mathrm{V}$ ) for 30 seconds followed by immersion in aqueous sodium hydro chlorite $(5 \% \mathrm{v} / \mathrm{v})$ for 20 minutes to prevent contamination and then washed six times with distilled water. For germination parameters seeds were sown in petri dishes and for seedling parameters seeds were sown in trays with soil after sterilization and kept in BOD germinator regulated to $28^{\circ} \mathrm{C}$ for the control, $13^{\circ} \mathrm{C}$ and $8^{\circ} \mathrm{C}$ for the cold treatment. The experiment was conducted in a randomized complete block design with three replicates considering each rack in the incubator as one block. Utilizing the mean data on root and shoot length $(\mathrm{cm})$, seedling length $(\mathrm{cm})$, the characters germination (\%), germination index, and seedling vigour index were calculated. $\boldsymbol{t}$-test was performed to compare the significance of mean differences of germination and seedling traits for normal vs $13^{\circ} \mathrm{C}$ and normal vs $8^{\circ} \mathrm{C}$ by using WINDOWSTAT software. The calculated value was compared with ' $t$ ' tabulated value at 0.05 significance.

\section{Results and Discussion}

The study of per se performance indicated that cold tolerance was related physiological parameters like germination percent, germination Index, root, shoot and seedling length, and seedling vigour. These parameters were chosen as they were the most adequate to differentiate cold tolerance exhibited by cold resistance genotypes at the germination stage (Cruz and Milach, 2004). It is very crucial to have a better understanding of the mechanism of cold tolerance at seedling stage, since the rice crop grown during rabi/boro season is affected by cold stress during nursery stage. 
The mean values and percent reduction of genotypes at different temperatures for various traits are presented in tables 1-3. The response of genotypes differed with different cold temperatures. There was considerable variability for cold tolerance at the germination stage among the genotypes. Cruz and Milach (2004), Sharifi Peyman and Aminpanah Hashem (2010) and Carolina et al., (2013) studied under different cold temperatures and reported similar results. Germination (\%) ranged from 60 to $100 \%$ at normal temperature. In case of $13^{\circ} \mathrm{C}$ germination $(\%)$ ranged from 50.0 to 100 and at $8^{\circ} \mathrm{C}$ it ranged from 53.3 (China-988) to 90.0(HPR-2336). The percent of reduction in mean germination was 1.8 and 17.6 at $13^{\circ} \mathrm{C}$ and $8^{\circ} \mathrm{C}$, respectively (Table 1) as compared to normal temperature. As many as 16 genotypes recorded significantly superior germination percent with very less reduction at $13^{\circ} \mathrm{C}$. However, at $8^{\circ} \mathrm{C}$ only 8 genotypes showed significantly superior germination percentage. The genotypes SKAU- 382, SKAU-5, SKAU-389, HPR-1068, HPR- 2336 and BPT 5204 registered significantly superior germination (\%) along with very low percentage of reduction at both temperatures. Germination (\%) seems to be the most appropriate trait to evaluate cold tolerance during seed germination period (Cruz and Milach, 2004 and Priyanka et al., 2015). Similarly, germination index (GI) expresses the germination speed under cold temperature. GI calculated based on seeds that presented coleoptiles and radical and is an important index for better performance of genotypes under the cold stress. The genotypes China-1007 (56.7, 48.3) and RP2421 and Vivek Dhan $82(100,100)$ recorded lowest and highest germination index at normal and $13^{\circ} \mathrm{C}$ respectively. However, the range was from 41.7 (China -988) to 83.3 (HPR-2336) at $8^{\circ} \mathrm{C}$. At $13^{\circ} \mathrm{C}$ reduction in germination Index (\%) was very low for most of the genotypes and four genotypes, China
1007, HPR 2336, K-475 and HPR 1068 showed less than $20 \%$ reduction at and $8^{\circ} \mathrm{C}$ (Table 1). These results indicated that more number of seeds of these genotypes had germinated at 14 and 21 days after the sowing. In fact, variation in environmental effects is one of the limiting factors against evaluation of germination, in order to identify genetically best lines for cold stress conditions (Cruz et al., 2006). In order to enhance and ensure uniform establishment of rice seedlings in the early season, cold tolerance during this time is vital (Sato et al., 2001).

Root and shoot length and Seedling growth are the important traits and genotypes showing less reduction percentage for these traits show cold tolerance. The genotypes China- $1007(10.7 \mathrm{~cm})$, Chenab $(7.6 \mathrm{~cm})$ and Vivek Dhan-82 $(3.3 \mathrm{~cm})$ recorded highest root length at normal, $8^{\circ} \mathrm{C}$ and $13^{\circ} \mathrm{C}$ respectively. The genotype Himalaya-741 registered lowest root length at normal $(7.2 \mathrm{~cm})$ and $13^{\circ} \mathrm{C}(3.5$ $\mathrm{cm})$. Shoot length ranged from $5.4 \mathrm{~cm}$ (Himalaya-1) to $12.1 \mathrm{~cm}$ (SKAU-382) at normal temperature. Shoot length ranged from $3.5 \mathrm{~cm}$ (Himalaya-2216) to $8.4 \mathrm{~cm}$ (Chenab) at $13^{\circ} \mathrm{C}$ and $1.1 \mathrm{~cm}$ (MTU 1010) to $2.9 \mathrm{~cm}$ (Vivek Dhan -82 at $8^{\circ} \mathrm{C}$. Genotypes SKU-382, SKAU-5, Chenab, Sukardhan-1, HPR-2336, Vivek Dhan -65, Vivek Dhan -86 and Vivek Dhan -206 recorded significantly superior performance over the population mean for root and shoot length and seedling growth. The genotypes Chenab, Sukardhan-1, HPR-2336, and Vivek Dhan -86 recorded lesser than 25 percent of reduction for the root, shoot length and seedling growth at $13^{\circ} \mathrm{C}$. At $8^{\circ} \mathrm{C}$ genotypes Himalaya-1, Himalaya -2216, HPR 1068, HPR-2336, Vivek Dhan-82 and Vivek Dhan-65 were significantly superior over the mean and recorded lesser than 75 percent of reduction for the root, shoot length and seedling growth table. 
Table.1 Mean values and percent reduction of rice genotypes for germination and Germination Index at low temperature regimes

\begin{tabular}{|c|c|c|c|c|c|c|c|c|c|c|}
\hline \multirow[b]{2}{*}{ Entry } & \multicolumn{5}{|c|}{ Germination $(\%)$} & \multicolumn{5}{|c|}{ Germination Index } \\
\hline & $\mathrm{N}$ & $13^{\circ} \mathrm{C}$ & $\begin{array}{l}\% \\
\text { reduction } \\
13^{\circ} \mathrm{C}\end{array}$ & $8^{\circ} \mathrm{C}$ & $\begin{array}{l}\% \\
\text { reduction } \\
8^{\circ} \mathrm{C} \\
\end{array}$ & $\mathrm{N}$ & $13^{\circ} \mathrm{C}$ & $\begin{array}{l}\% \\
\text { reduction } \\
13^{\circ} \mathrm{C}\end{array}$ & 8 & $\begin{array}{l}\% \\
\text { reduction } \\
8^{\circ} \mathrm{C} \\
\end{array}$ \\
\hline China-1039 & 80.0 & 86.7 & -8.4 & 76.7 & 4.1 & 73.3 & 81.7 & -11.5 & 56.7 & 30.6 \\
\hline SKAU-382 & $100.0^{*}$ & $100.0 *$ & 0.0 & $83.3^{*}$ & 16.7 & $98.3^{*}$ & $98.3^{*}$ & 0.0 & 63.3 & 35.6 \\
\hline $\mathrm{K}-475$ & 80.0 & 66.7 & 16.6 & 63.3 & 20.9 & 60.0 & 51.7 & 13.8 & 50.0 & 3.3 \\
\hline China-988 & 70.0 & 63.3 & 9.6 & 53.3 & 23.9 & 63.3 & 58.3 & 7.9 & 41.7 & 28.5 \\
\hline Jhelum & 86.7 & 86.7 & 0.0 & 80.0 & 7.7 & 85.0 & 86.7 & -2.0 & $66.7 *$ & 23.1 \\
\hline SKAU-5 & $100.0 *$ & $96.7 *$ & 3.3 & 76.7 & 23.3 & $98.3 *$ & $96.7 *$ & 1.6 & 60.0 & 38.0 \\
\hline China-1007 & 60.0 & 50.0 & 16.7 & 66.7 & -11.2 & 56.7 & 48.3 & 14.8 & 51.7 & -7.0 \\
\hline Shalimar-1 & 90.0 & 90.0 & 0.0 & $83.3 *$ & 7.4 & 85.0 & 86.7 & -2.0 & $66.7 *$ & 23.1 \\
\hline SKAU-389 & $100.0 *$ & 93.3 & 6.7 & $86.7 *$ & 13.3 & $98.3 *$ & 91.7 & 6.7 & 65.0 & 29.1 \\
\hline Chenab & 86.7 & 86.7 & 0.0 & 73.3 & 15.5 & 83.3 & 85.0 & -2.0 & 58.3 & 31.4 \\
\hline K-332 & 66.7 & 70.0 & -4.9 & 16.7 & 75.0 & 58.3 & 58.3 & 0.0 & 13.3 & 77.2 \\
\hline SKAU-339 & $100.0 *$ & $96.7 *$ & 3.3 & 80.0 & 20.0 & $91.7 *$ & 91.7 & 0.0 & 63.3 & 31.0 \\
\hline SKAU-341 & $96.7 *$ & 93.3 & 3.5 & 76.7 & 20.7 & $95.0 *$ & 91.7 & 3.5 & 60.0 & 34.6 \\
\hline K-429 & 90.0 & 90.0 & 0.0 & 66.7 & 25.9 & 76.7 & 76.7 & 0.0 & 50.0 & 34.8 \\
\hline Himalaya-1 & 93.3 & 93.3 & 0.0 & 76.7 & 17.8 & 88.3 & 90.0 & -1.9 & 63.3 & 29.7 \\
\hline Himalaya-741 & $100.0 *$ & $96.7 *$ & 3.3 & 76.7 & 23.3 & $100.0 *$ & $96.7 *$ & 3.3 & 65.0 & 32.8 \\
\hline Himalaya-2216 & 93.3 & $96.7 *$ & -3.6 & 76.7 & 17.8 & $93.3 *$ & $96.7 *$ & -3.6 & 60.0 & 38.0 \\
\hline RP-2421 & $100.0 *$ & $100.0 *$ & 0.0 & 80.0 & 20.0 & $100.0 *$ & $100.0^{*}$ & 0.0 & 63.3 & 36.7 \\
\hline HPR-2143 & 86.7 & 90.0 & -3.8 & 80.0 & 7.7 & 85.0 & 90.0 & -5.9 & 65.0 & 27.8 \\
\hline HPR-1068 & 93.3 & $96.7 *$ & -3.6 & $86.7 *$ & 7.1 & 90.0 & $93.3 *$ & -3.7 & $71.7 *$ & 23.2 \\
\hline Sukaradhan-1 & 93.3 & 90.0 & 3.5 & $86.7 *$ & 7.1 & 91.7 & 90.0 & 1.9 & $70.0 *$ & 22.2 \\
\hline HPR-2373 & 93.3 & 83.3 & 10.7 & 66.7 & 28.5 & $93.3 *$ & 81.7 & 12.4 & 53.3 & 34.8 \\
\hline HPR-2336 & 96.7 & $96.7 *$ & 0.0 & $90.0^{*}$ & 6.9 & $96.7 *$ & $96.7 *$ & 0.0 & $83.3 *$ & 13.9 \\
\hline HPR-2513 & 83.3 & 76.7 & 7.9 & 70.0 & 16.0 & 81.7 & 75.0 & 8.2 & 55.0 & 26.7 \\
\hline Vivek Dhan-85 & $100.0 *$ & $100.0 *$ & 0.0 & 76.7 & 23.3 & $98.3 *$ & $98.3 *$ & 0.0 & 61.7 & 37.2 \\
\hline Vivek Dhan -82 & $100.0^{*}$ & $100.0^{*}$ & 0.0 & 66.7 & 33.3 & $100.0 *$ & $100.0 *$ & 0.0 & 50.0 & 50.0 \\
\hline Vivek Dhan -62 & 93.3 & 93.3 & 0.0 & $83.3 *$ & 10.7 & 90.0 & 88.3 & 1.9 & $68.3^{*}$ & 22.7 \\
\hline Vivek Dhan -65 & $100.0 *$ & $100.0 *$ & 0.0 & 80.0 & 20.0 & $96.7 *$ & $98.3 *$ & -1.7 & $66.7 *$ & 32.1 \\
\hline V L Dhan-86 & 93.3 & 93.3 & 0.0 & 73.3 & 21.4 & 90.0 & 91.7 & -1.9 & 53.3 & 41.9 \\
\hline V L Dhan-206 & $100.0 *$ & $100.0 *$ & 0.0 & 76.7 & 23.3 & $100.0 *$ & $98.3 *$ & 1.7 & 60.0 & 39.0 \\
\hline V L Dhan -207 & $100.0^{*}$ & $100.0^{*}$ & 0.0 & 80.0 & 20.0 & $100.0 *$ & $98.3^{*}$ & 1.7 & 65.0 & 33.9 \\
\hline V L Dhan -208 & $100.0 *$ & $96.7 *$ & 3.3 & 80.0 & 20.0 & $100.0^{*}$ & $96.7 *$ & 3.3 & $68.3^{*}$ & 29.4 \\
\hline V L Dhan -209 & 93.3 & $96.7 *$ & -3.6 & 76.7 & 17.8 & 91.7 & 90.0 & 1.9 & 56.7 & 37.0 \\
\hline MTU 1010 & 93.3 & 90.0 & 3.5 & 80.0 & 14.3 & 90.0 & 88.3 & 1.9 & 61.7 & 30.1 \\
\hline Tellahamsa & 73.3 & 66.7 & 9.0 & 70.0 & 4.5 & 70.0 & 65.0 & 7.1 & 53.3 & 18.0 \\
\hline Rajendra & 76.7 & 73.3 & 4.4 & 66.7 & 13.0 & 71.7 & 68.3 & 4.7 & 55.0 & 19.5 \\
\hline BPT 5204 & $96.7 *$ & $100.0^{*}$ & -3.4 & $83.3^{*}$ & 13.9 & $95.0 *$ & $98.3^{*}$ & -3.5 & $70.0^{*}$ & 28.8 \\
\hline Mean & 90.8 & 89.2 & & 74.8 & & 87.5 & 86.3 & & 59.6 & \\
\hline CD\% & & 5.32 & & 5.33 & & & 6.27 & & 5.55 & \\
\hline
\end{tabular}


Table.2 The mean values and percent reduction of rice genotypes for different temperatures for root and shoot length

\begin{tabular}{|c|c|c|c|c|c|c|c|c|c|c|}
\hline \multirow[b]{2}{*}{ Entry } & \multicolumn{5}{|c|}{ Root length } & \multicolumn{5}{|c|}{ Shoot length } \\
\hline & $\mathrm{N}$ & $13^{\circ} \mathrm{C}$ & $\begin{array}{l}\% \\
\text { reduction } \\
13^{\circ} \mathrm{C}\end{array}$ & $8^{\circ} \mathrm{C}$ & $\begin{array}{l}\% \\
\text { reduction } \\
8^{\circ} \mathrm{C}\end{array}$ & $\mathrm{N}$ & $13^{\circ} \mathrm{C}$ & $\begin{array}{l}\% \\
\text { reduction } \\
13^{\circ} \mathrm{C}\end{array}$ & $8^{\circ} \mathrm{C}$ & $\begin{array}{l}\% \\
\text { reduction } \\
8^{\circ} \mathrm{C}\end{array}$ \\
\hline China-1039 & 8.1 & 4.8 & 40.7 & 1.4 & 82.7 & 7.1 & 4.7 & 33.8 & 1.4 & 80.3 \\
\hline SKAU-382 & 8.3 & $6.8^{*}$ & 18.1 & 1.9 & 77.1 & 12.1 & $6.5^{*}$ & 46.3 & 1.6 & 86.8 \\
\hline K-475 & 8.3 & 6.3 & 24.1 & 1.7 & 79.5 & 7.4 & 5.8 & 21.6 & 1.6 & 78.4 \\
\hline China-988 & 7.4 & 6.0 & 18.9 & 1.9 & 74.3 & 11.8 & 5.6 & 52.5 & 1.8 & 84.7 \\
\hline Jhelum & 8.4 & $7.3^{*}$ & 13.1 & 2.1 & 75.0 & 7.9 & 5.6 & 29.1 & 1.8 & 77.2 \\
\hline SKAU-5 & $9.8^{*}$ & $6.8^{*}$ & 30.6 & 2.9 & 70.4 & 7.6 & 5.9 & 22.4 & 2.4 & 68.4 \\
\hline China-1007 & $10.7^{*}$ & $6.7 *$ & 37.4 & 2.0 & 81.3 & 9.6 & $7.3^{*}$ & 24.0 & 1.8 & 81.3 \\
\hline Shalimar-1 & $9.8^{*}$ & $6.9^{*}$ & 29.6 & 1.9 & 80.6 & 7.6 & 5.8 & 23.7 & 1.7 & 77.6 \\
\hline SKAU-389 & 8.9 & $7.1 *$ & 20.2 & 2.1 & 76.4 & 9.3 & 6.1 & 34.4 & 1.4 & 84.9 \\
\hline Chenab & 8.7 & $7.6^{*}$ & 12.6 & 1.7 & 80.5 & 10.6 & $8.4^{*}$ & 20.8 & 1.7 & 84.0 \\
\hline K-332 & 7.9 & 4.3 & 45.6 & 1.7 & 78.5 & 5.7 & 4.3 & 24.6 & 1.4 & 75.4 \\
\hline SKAU-339 & 8.1 & 4.3 & 46.9 & 2.0 & 75.3 & 9.1 & 4.9 & 46.2 & 1.9 & 79.1 \\
\hline SKAU-341 & 8.4 & 4.5 & 46.4 & 1.3 & 84.5 & 5.6 & 4.6 & 17.9 & 1.3 & 76.8 \\
\hline K-429 & 8.7 & 5.1 & 41.4 & 2.3 & 73.6 & 7.8 & 5.6 & 28.2 & 1.9 & 75.6 \\
\hline Himalaya-1 & 8.1 & 5.8 & 28.4 & 2.5 & 69.1 & 5.4 & 4.5 & 16.7 & $2.5^{*}$ & 53.7 \\
\hline Himalaya-741 & 7.2 & 3.5 & 51.4 & 1.5 & 79.2 & 6.8 & 3.7 & 45.6 & 1.4 & 79.4 \\
\hline Himalaya-2216 & 7.9 & 3.8 & 51.9 & 2.7 & 65.8 & 7.8 & 3.5 & 55.1 & $2.5^{*}$ & 67.9 \\
\hline RP-2421 & 8.1 & 5.8 & 28.4 & 2.4 & 70.4 & 9.3 & 4.9 & 47.3 & 2.1 & 77.4 \\
\hline HPR-2143 & 7.6 & 5.8 & 23.7 & 2.4 & 68.4 & 8.6 & 5.2 & 39.5 & 2.2 & 74.4 \\
\hline HPR-1068 & $10.4^{*}$ & $6.4^{*}$ & 38.5 & 3.2 & 69.2 & 9.1 & 5.5 & 39.6 & $2.8^{*}$ & 69.2 \\
\hline Sukaradhan-1 & $9.1^{*}$ & $7.2^{*}$ & 20.9 & 2.5 & 72.5 & 9.6 & $8.3^{*}$ & 13.5 & 1.8 & 81.3 \\
\hline HPR-2373 & 7.3 & 5.9 & 19.2 & 2.0 & 72.6 & 5.7 & $6.9 *$ & -21.1 & 1.5 & 73.7 \\
\hline HPR-2336 & $9.4^{*}$ & $7.3^{*}$ & 22.3 & 2.8 & 70.2 & 11.3 & $7.1^{*}$ & 37.2 & $2.6^{*}$ & 77.0 \\
\hline HPR-2513 & 8.2 & 5.3 & 35.4 & 1.8 & 78.0 & 6.7 & 4.9 & 26.9 & 1.8 & 73.1 \\
\hline Vivek Dhan-85 & 7.9 & 4.3 & 45.6 & 2.0 & 74.7 & 6.4 & 3.8 & 40.6 & 1.7 & 73.4 \\
\hline Vivek Dhan -82 & 8.9 & 5.9 & 33.7 & 3.3 & 62.9 & 8.1 & $6.9^{*}$ & 14.8 & $2.9^{*}$ & 64.2 \\
\hline Vivek Dhan -62 & $9.1^{*}$ & 5.4 & 40.7 & 1.8 & 80.2 & 8.3 & 5.1 & 38.6 & 1.7 & 79.5 \\
\hline Vivek Dhan -65 & $9.3^{*}$ & 5.7 & 38.7 & 2.6 & 72.0 & 8.9 & $6.9 *$ & 22.5 & 2.3 & 74.2 \\
\hline V L Dhan-86 & $9.8^{*}$ & $7.6^{*}$ & 22.4 & 2.2 & 77.6 & 10.2 & $8.3^{*}$ & 18.6 & 1.9 & 81.4 \\
\hline V L Dhan-206 & 9.0 & $6.3^{*}$ & 30.0 & 2.1 & 76.7 & 6.5 & 5.3 & 18.5 & 2.0 & 69.2 \\
\hline V L Dhan -207 & 7.7 & 3.8 & 50.6 & 2.2 & 71.4 & 5.5 & 3.8 & 30.9 & 2.2 & 60.0 \\
\hline V L Dhan -208 & 8.3 & $6.4^{*}$ & 22.9 & 2.1 & 74.7 & 7.8 & 5.4 & 30.8 & 1.9 & 75.6 \\
\hline V L Dhan -209 & 8.9 & 4.7 & 47.2 & 1.8 & 79.8 & 5.4 & 3.8 & 29.6 & 1.6 & 70.4 \\
\hline MTU 1010 & $9.9 *$ & 5.9 & 40.4 & 1.4 & 85.9 & 5.7 & 4.3 & 24.6 & 1.1 & 80.7 \\
\hline Tellahamsa & $9.4^{*}$ & 5.3 & 43.6 & 1.7 & 81.9 & 7.9 & 4.8 & 39.2 & 1.7 & 78.5 \\
\hline Rajendra & $9.8^{*}$ & 5.9 & 39.8 & 1.2 & 87.8 & 5.9 & 4.3 & 27.1 & 1.1 & 81.4 \\
\hline BPT 5204 & 8.9 & 3.7 & 58.4 & 1.3 & 85.4 & 6.5 & 3.6 & 44.6 & 1.3 & 80.0 \\
\hline Mean & 8.7 & 5.7 & 34.5 & 2.1 & 75.9 & 7.9 & 5.5 & 30.4 & 1.9 & 75.9 \\
\hline CD\% & & 0.48 & & 0.33 & & & 0.75 & & 0.63 & \\
\hline
\end{tabular}


Table.3 The mean values and percent reduction of rice genotypes for different temperatures for seedling growth and Seedling Vigour Index I

\begin{tabular}{|c|c|c|c|c|c|c|c|c|c|c|}
\hline \multirow[b]{2}{*}{ Entry } & \multicolumn{5}{|c|}{ Seedling growth } & \multicolumn{5}{|c|}{ Seedling vigour } \\
\hline & $\mathrm{N}$ & $13^{\circ} \mathrm{C}$ & $\begin{array}{l}\% \\
\text { reduction } \\
13^{\circ} \mathrm{C} \\
\end{array}$ & $8^{\circ} \mathrm{C}$ & $\begin{array}{l}\% \\
\text { reduction } \\
8^{\circ} \mathrm{C} \\
\end{array}$ & $\mathrm{N}$ & $13^{\circ} \mathrm{C}$ & $\begin{array}{l}\% \\
\text { reduction } \\
13^{\circ} \mathrm{C}\end{array}$ & $8^{\circ} \mathrm{C}$ & $\begin{array}{l}\% \\
\text { reduction } \\
8^{\circ} \mathrm{C}\end{array}$ \\
\hline China-1039 & 15.3 & 9.5 & 37.9 & 2.8 & 81.7 & 1222.9 & 826.6 & 32.4 & 214.9 & 82.4 \\
\hline SKAU-382 & 20.5 & $13.3^{*}$ & 35.1 & 3.5 & 82.9 & $2045.3 *$ & $1328.0 *$ & 35.1 & 289.3 & 85.9 \\
\hline K-475 & 15.7 & 12.2 & 22.3 & 3.4 & 78.3 & 1255.1 & 810.6 & 35.4 & 213.9 & 83.0 \\
\hline China-988 & 19.3 & 11.7 & 39.4 & 3.8 & 80.3 & 1348.1 & 739.2 & 45.2 & 200.2 & 85.1 \\
\hline Jhelum & 16.3 & $12.9^{*}$ & 20.9 & 3.9 & 76.1 & 1412.0 & $1118.5^{*}$ & 20.8 & 312.0 & 77.9 \\
\hline SKAU-5 & 17.4 & $12.7^{*}$ & 27.0 & $5.3^{*}$ & 69.5 & $1743.3^{*}$ & $1230.9^{*}$ & 29.4 & $404.0^{*}$ & 76.8 \\
\hline China-1007 & 20.3 & $14.1^{*}$ & 30.5 & 3.8 & 81.3 & 1220.4 & 703.8 & 42.3 & 251.0 & 79.4 \\
\hline Shalimar-1 & 17.5 & $12.8^{*}$ & 26.9 & 3.7 & 78.9 & $1572.6^{*}$ & $1149.9^{*}$ & 26.9 & 304.6 & 80.6 \\
\hline SKAU-389 & 18.3 & $13.2^{*}$ & 27.9 & 3.5 & 80.9 & $1827.3^{*}$ & $1234.1^{*}$ & 32.5 & 301.0 & 83.5 \\
\hline Chenab & 19.4 & $16.1^{*}$ & 17.0 & 3.4 & 82.5 & $1679.8^{*}$ & 1392.4* & 17.1 & 251.1 & 85.1 \\
\hline K-332 & 13.7 & 8.6 & 37.2 & 3.1 & 77.4 & 911.0 & 602.8 & 33.8 & 52.3 & 94.3 \\
\hline SKAU-339 & 17.3 & 9.2 & 46.8 & 4.0 & 76.9 & $1728.0 *$ & 886.5 & 48.7 & 317.6 & 81.6 \\
\hline SKAU-341 & 14.0 & 9.1 & 35.0 & 2.6 & 81.4 & 1357.7 & 853.2 & 37.2 & 198.4 & 85.4 \\
\hline K-429 & 16.6 & 10.7 & 35.5 & 4.2 & 74.7 & 1492.8 & 964.8 & 35.4 & 279.8 & 81.3 \\
\hline Himalaya-1 & 13.4 & 10.3 & 23.1 & $5.0^{*}$ & 62.7 & 1253.2 & 961.6 & 23.3 & 382.6 & 69.5 \\
\hline Himalaya-741 & 14.0 & 7.2 & 48.6 & 2.9 & 79.3 & 1399.3 & 691.9 & 50.6 & 218.3 & 84.4 \\
\hline Himalaya-2216 & 15.8 & 7.3 & 53.8 & $5.2^{*}$ & 67.1 & 1474.1 & 706.6 & 52.1 & $395.8 *$ & 73.1 \\
\hline RP-2421 & 17.3 & 10.8 & 37.6 & 4.6 & 73.4 & $1730.3^{*}$ & 1076.3 & 37.8 & 367.0 & 78.8 \\
\hline HPR-2143 & 16.2 & 11.0 & 32.1 & $4.7^{*}$ & 71.0 & 1404.1 & 989.7 & 29.5 & 372.3 & 73.5 \\
\hline HPR-1068 & 19.5 & 11.9 & 39.0 & $6.1^{*}$ & 68.7 & $1819.9^{*}$ & $1147.9^{*}$ & 36.9 & $527.0^{*}$ & 71.0 \\
\hline Sukaradhan-1 & 18.7 & $15.5^{*}$ & 17.1 & 4.3 & 77.0 & $1743.7^{*}$ & $1396.2^{*}$ & 19.9 & 371.8 & 78.7 \\
\hline HPR-2373 & 13.0 & $12.8^{*}$ & 1.5 & 3.5 & 73.1 & 1212.9 & 1062.9 & 12.4 & 235.3 & 80.6 \\
\hline HPR-2336 & 20.7 & $14.5^{*}$ & 30.0 & $5.5^{*}$ & 73.4 & $2002.0 *$ & $1399.2^{*}$ & 30.1 & $493.0 *$ & 75.4 \\
\hline HPR-2513 & 15.0 & 10.2 & 32.0 & 3.6 & 76.0 & 1246.1 & 778.0 & 37.6 & 251.6 & 79.8 \\
\hline Vivek Dhan-85 & 14.4 & 8.1 & 43.8 & 3.7 & 74.3 & 1436.7 & 805.0 & 44.0 & 282.1 & 80.4 \\
\hline Vivek Dhan -82 & 17.1 & $12.9^{*}$ & 24.6 & $6.2 *$ & 63.7 & $1708.7 *$ & $1285.0 *$ & 24.8 & $416.0 *$ & 75.7 \\
\hline Vivek Dhan -62 & 17.5 & 10.5 & 40.0 & 3.4 & 80.6 & $1630.6^{*}$ & 984.8 & 39.6 & 284.9 & 82.5 \\
\hline Vivek Dhan -65 & 18.2 & $12.6^{*}$ & 30.8 & $4.8^{*}$ & 73.6 & $1819.7 *$ & $1264.3^{*}$ & 30.5 & $385.5^{*}$ & 78.8 \\
\hline V L Dhan-86 & 20.0 & $15.9^{*}$ & 20.5 & 4.2 & 79.0 & $1868.7 *$ & $1486.6^{*}$ & 20.4 & 305.3 & 83.7 \\
\hline V L Dhan-206 & 15.5 & 11.6 & 25.2 & 4.0 & 74.2 & 1551.0 & $1159.3^{*}$ & 25.3 & 308.8 & 80.1 \\
\hline V L Dhan -207 & 13.3 & 7.6 & 42.9 & 4.4 & 66.9 & 1326.7 & 763.0 & 42.5 & 351.2 & 73.5 \\
\hline V L Dhan -208 & 16.1 & 11.8 & 26.7 & 4.0 & 75.2 & $1614.0 *$ & $1139.7 *$ & 29.4 & 317.5 & 80.3 \\
\hline V L Dhan -209 & 14.2 & 8.5 & 40.1 & 3.4 & 76.1 & 1329.1 & 820.4 & 38.3 & 257.0 & 80.7 \\
\hline MTU 1010 & 15.7 & 10.2 & 35.0 & 2.5 & 84.1 & 1463.1 & 920.4 & 37.1 & 200.0 & 86.3 \\
\hline Tellahamsa & 17.2 & 10.2 & 40.7 & 3.4 & 80.2 & 1264.0 & 678.2 & 46.3 & 236.1 & 81.3 \\
\hline Rajendra & 15.7 & 10.2 & 35.0 & 2.3 & 85.4 & 1205.3 & 748.7 & 37.9 & 154.1 & 87.2 \\
\hline BPT 5204 & 15.4 & 7.3 & 52.6 & 2.6 & 83.1 & 1488.5 & 727.3 & 51.1 & 215.5 & 85.5 \\
\hline Mean & 16.6 & 11.2 & 32.5 & 3.9 & 76.5 & 1508.3 & 995.5 & 34.0 & 295.1 & 80.4 \\
\hline CD\% & & 1.07 & & 0.78 & & & 116.98 & & 89.64 & \\
\hline
\end{tabular}


Seedling vigour index is an important parameter to assess the establishment efficiency of a genotype. Out of the 37 genotypes, fourteen genotypes exhibited significant superior performance for this trait and the genotypes VL Dhan-86, Sukardhan-1, HPR-2336, Chenab and SKAU-382 recorded highest seedling vigour index at $13^{\circ} \mathrm{C}$ (table 3). Genotypes viz., SKAU-5, Himalaya 2216, HPR 1068, HPR-2336, Vivek Dhan -86 and Vivek Dhan -65 were identified as superior at $8^{\circ} \mathrm{C}$. HPR- 2373, Chenab, Sukardhan-1, and VL Dhan-86 showed only 20 percent reduction at $13^{\circ} \mathrm{C}$ and more than 80 percent reduction at $8^{\circ} \mathrm{C}$. At $8^{\circ} \mathrm{C}$ genotypes Himalaya 1, HPR 1068, Himalaya -2216, HPR-2143 and VL Dhan-207 recorded more than 75 percent reduction for seedling vigour Index. Over all, HPR 1068, Himalaya 2216 and Vivek Dhan -82 recorded lesser percentage of reduction at both the temperatures for seedling vigour index under controlled conditions and these genotypes can be used as cold tolerant donors. Massardo et al., (2000) reported evaluation of rice seed germination rate and new plant traits under more than one temperature treatments are necessary to distinguish cold tolerant genotypes from cold susceptible genotypes. Changrong et al., (2008) reported that the seeds from the few varieties could germinate quickly at low temperature but, the seedling growth was severely delayed by low temperature stress. He also reported that cold tolerance at germination and seedling stage were correlated.

This study revealed that traits at the early growth stage of the investigated rice genotypes were significantly influenced by low temperature stress. However, the response of these genotypes to low temperatures was significant. Certain genotypes performed very well under $13^{\circ} \mathrm{C}$ and $8^{\circ} \mathrm{C}$ for various seedling traits. However, genotypes which are performing well under $13^{\circ} \mathrm{C}$ did not perform well under $8^{\circ} \mathrm{C}$ of temperatures and vice versa. The genotypes Chenab, Sukardhan-1, HPR-2336, and Vivek Dhan -86 recorded less than 25 percent of reduction at $13^{\circ} \mathrm{C}$ for the seedling characters studied. However, at $8^{\circ} \mathrm{C}$ of temperature, genotypes Himalaya-1, Himalaya -2216, HPR 1068, HPR-2336, Vivek Dhan-82 and Vivek dhan-65 recorded less than 75 percent of reduction and were significantly superior to the mean. Based on the percent of reduction of seedling vigour traits, these genotypes can be identified as superior cold tolerant lines and may be exploited as tolerant source in future rice breeding programmes aimed at development of cold tolerant varieties.

\section{References}

Caroline Borges Bevilacqua, Daisy Ramirez Monzon, Eduardo Venske, Supratim Basu and Paulo Dejalma Zimmer, 2013. Application of Stress Indices for Low Temperature and Deep Sowing Stress Screening of Rice Genotypes. Pak J of Bio Sci, 16: 1618-1622.

Changrong Ye, Shu Fukai, Russell Reinke, Ian Godwin, Peter Snell and Jaya Basnayake. 2008. Screening rice genetic resources for cold tolerance at different growth stages. Global Issues Paddock Action. Proceedings of the 14th Australian Agronomy Conference. September 2008, Adelaide South Australia.

Cruz RP and Milach SCK..2013. Avoiding damage and achieving cold tolerance in rice plants. Food and energy security. 2(2): 96-119.

Cruz RP, Milach SCK and Luiz CF. 2006. Inheritance of rice cold tolerance at the germination stage. Genet.Mole.Biol, 29: 314-320.

Cruz. RP and Milach SCK. 2004. Cold to tolerance at the germination stage of rice: Methods of evaluation and 
characterization of genotypes. Sci. Agric. 61: 1-8.

Farzin PD, Mohammad KH and Masoud E. 2013. Methods for rice genotypes cold tolerance evaluation at germination stage. Int.j. agric. crop sci. 5-18: 21112116.

Massardo F, Corcuera L and Alberdi M. (2000). Embyro Pysiological responces to cold by two cultivars of oat during germination. Crop Sci., 40: 1694-1701.

Pathak AK, Pathak PK, Sharma KK (2003). Recent Development in Boro Rice Improvement. and Production for Raising Rice Yield in Assam. Boro Rice. Edi. Singh RK, Hossain M and Thakur R Intl. Rice Res. Inst. India Office, Pusa Campus, New Delhi, India, pp.73-80.

Priyanka K, Jaiswal HK S A, Waza and Sravan T. 2015. Response of rice seedlings to cold tolerance under boro conditions. SABRAO J. 47(2): 185-190.

Sato Y, Murakami T, Funatsuki H, Matsuba S, Saruyama H. 2001. Heat shockmediated APX gene expression and protection against chilling injury in rice seedlings. J. Exp. Bot. 52: 145-151.

Sharifi P, and Aminpanah H. 2010. Evaluation eighteen rice genotypes in cold tolerance at germination stage. World Appl Sci J. 11:1476-1480.

Sharifi Peyman and Aminpanah Hashem.2010. Evauation of eighteen rice genotypes in cold tolarence at germination stage. World Appl Sci J. 11(11): 1476-1480.

Tiwari V, Rautaray SK, Singh UD (2009). Response of rice genotypes to cold temperature in boro season. Rice Genetics Newsletter. Vol. 25.

\section{How to cite this article:}

Srikanth Rahul, N., D. Bhadru, M. Sreedhar and Vanisri, S. 2017. Screening of Cold Tolerant Rice Genotypes for Seedling Traits under Low Temperature Regimes. Int.J.Curr.Microbiol.App.Sci. 6(12): 4074-4081. doi: https://doi.org/10.20546/ijcmas.2017.612.468 